P2-280 THE EFFECTS OF SCOPOLAMINE ON SOCIABILITY AND SOCIAL MEMORY IN MICE: REVERSAL BY DONEPEZIL

Gernot Riedel, Bettina Platt, Aberdeen University, Aberdeen, United Kingdom. Contact e-mail: g.riedel@abdn.ac.uk

Background: Impaired aspects of social behaviour are core symptoms of psychiatric disorders. In rodents, social recognition is reliant on cholinergic receptor activation and this situation is reminiscent of patients suffering from dementia such as Alzheimer's disease (AD), in which a reduction of cholinergic tone and reduced activation of muscarinic receptors is a consistent hallmark and social disengagement is a risk factor for cognitive decline. Here, we the effects of diazepam and scopolamine in a modified social interaction task and probed reversal of deficits by donepezil, the most common treatment in AD. Methods: The tasks follows the principles laid out by Moy et al (Genes Brain Behav. 3: 287; 2004) and involves the automatic measurement of interactions between a resident and one stranger mouse (sociability) for five minutes and, then after an interval (30s-10 min) an additional second stranger mouse (social memory). The time spent investigating the strangers is taken as an index of sociability and was conducted using group-housed female C57Bl/6 mice ( $\mathrm{n}=12$ per drug) that received i.p. administration of $0.3 \mathrm{mg} / \mathrm{kg}$ scopolamine with and without donepezil $(1 \mathrm{mg} / \mathrm{kg}$ ) or $0.1 \mathrm{mg} / \mathrm{kg}$ diazepam or vehicle (saline). Results: All treatment groups showed sociability. However, while both the vehicle and diazepam treatment groups also displayed a significant social memory, this memory was absent in scopolamine-treated mice which only showed sociability, but no social memory. Donepezil co-treatment prevented the social memory deficit. Conclusions: These data suggest that the development of social memory or its recall is dependent on muscarinic, but not benzodiazepine receptor activation. Reversal of memory deficits by donepezil suggest that social recognition may be a useful test in $\mathrm{AD}$ and patients on treatment with acetylcholine inhibitors may considerably benefit and improve social behaviour.

P2-281 POLYPHARMACY AMONG COMMUNITYDWELLING ELDERLY PATIENTS WITH DEMENTIA: ANALYSIS OF THE NATIONAL ALZHEIMER'S COORDINATING CENTER UNIFORM DATA SET

Sandra Weintraub ${ }^{1}$, Nathaniel Mercaldo ${ }^{2}$, Andrew T. Harris ${ }^{3}$, Denys T. Lau ${ }^{3},{ }^{1}$ Northwestern University, Alzheimer's Disease Center, Chicago, IL, USA; ${ }^{2}$ University of Washington, National Alzheimer's Coordinating Center, Seattle, WA, USA; ${ }^{3}$ Northwestern University, Buehler Center on Aging, Health \& Society, Chicago, IL, USA. Contact e-mail: sweintraub@northwestern.edu

Background: Polypharmacy — the use of high number of medications-is often viewed as an indicator of overmedication. This study examines the prevalence and patient characteristics associated with polypharmacy among elderly patients with dementia. Methods: Data (9/2005-9/2007) were from the National Alzheimer's Coordinating Center Uniform Data Set, which contained information collected at 29 NIA-funded Alzheimer's Disease Centers across the U.S. This cross-sectional study analyzed 2,665 community-dwelling, dementia patients aged $65+$ who took at least one prescription medication. Polypharmacy was defined as taking $5+$ medications. Multivariate logistic regressions accounting for center effect were performed. Results: Among the study sample, the mean number of medications taken was $5.1(\mathrm{SD}=2.7)$, and $53.8 \%$ had polypharmacy $(28.3 \%$ took 5-6 medications; $14.7 \%$ took 7-8 medications; $10.8 \%$ took $9+$ medications). Among the primary diagnoses of dementia that had $30+$ cases, Parkinson's disease $(33 / 53=62 \%)$, vascular dementia $(21 / 40=53 \%)$, and dementia with Lewy bodies $(64 / 127=50 \%)$ had the highest proportion of patients with polypharmacy. Probable and possible Alzheimer's disease had 37\% (736/1975) and 43\% (108/252), respectively. Patient characteristics associated with higher odds of polypharmacy were age 75-79 $(\mathrm{OR}=1.43 ; 95 \% \mathrm{CI}=1.00-2.04)$ and age $80-84(\mathrm{OR}=1.46 ; 95 \% \mathrm{CI}=1.01$ -
$2.12)$ vs. age 65-74; cardiovascular disease $(\mathrm{OR}=1.70$; $95 \% \mathrm{CI}=1.15$ 2.52); mental disorder $(\mathrm{OR}=2.37$; $95 \% \mathrm{CI}=1.71-3.27)$; hypertension $(\mathrm{OR}=3.49 ; 95 \% \mathrm{CI}=2.80-4.35)$; hypercholesterolemia $(\mathrm{OR}=2.28 ; 95 \%$ $\mathrm{CI}=1.84-2.82)$; diabetes $(\mathrm{OR}=3.74 ; 95 \% \mathrm{CI}=2.55-5.49)$; thyroid disease $(\mathrm{OR}=2.81 ; 95 \% \mathrm{CI}=2.02-3.91)$; incontinence $(\mathrm{OR}=1.31 ; 95 \% \mathrm{CI}=1.01$ 1.69); appetite problems $(\mathrm{OR}=1.70$; $95 \% \mathrm{CI}=1.17-2.48)$; and use of medications that should be avoided among elderly patients as defined by Beers 2003 criteria $(\mathrm{OR}=3.69 ; 95 \% \mathrm{CI}=2.98-5.26)$. Characteristics associated with lower odds of polypharmacy were moderate/severe anxiety $(\mathrm{OR}=0.63 ; 95 \% \mathrm{CI}=0.44-0.88)$; and African-Americans $(\mathrm{OR}=0.60 ; 95 \%$ $\mathrm{CI}=0.43-0.85)$ vs. Caucasians. Analyses redefining polypharmacy as taking $9+$ medications found that cerebrovascular disease $(\mathrm{OR}=2.10 ; 95 \%$ $\mathrm{CI}=1.22-3.64)$; moderate/severe depression $(\mathrm{OR}=2.15$; $95 \% \mathrm{CI}=1.10$ 4.21); and mild $(\mathrm{OR}=1.96 ; 95 \% \mathrm{CI}=1.26-3.04)$ and moderate/severe nighttime behavior problems $(\mathrm{OR}=1.80 ; 95 \% \mathrm{CI}=1.13-2.88)$ also had higher odds of polypharmacy, while severe cognitive impairment $(\mathrm{CDR}=3)(\mathrm{OR}=0.26 ; 95 \% \mathrm{CI}=0.09-0.78)$; and mild $(\mathrm{OR}=0.56 ; 95 \%$ $\mathrm{CI}=0.35-0.88)$ and moderate/severe apathy $(\mathrm{OR}=0.63 ; 95 \% \mathrm{CI}=0.40-$ 0.99 ) had lower odds. Conclusions: Polypharmacy is common among community-dwelling elderly patients with dementia. Interventions should target at-risk patients. The impact of polypharmacy on this population needs further investigation.

\section{P2-282 IMPACT OF A CONDENSED PROTOCOL FOR DISCLOSING APOE GENOTYPE TO FIRST- DEGREE RELATIVES OF PEOPLE WITH ALZHEIMER'S DISEASE}

Scott Roberts ${ }^{1}$, Erin Linnenbringer ${ }^{1}$, Melissa Barber Butson ${ }^{2}$, Peter Whitehouse ${ }^{2}$, Grace Fasaye ${ }^{3}$, Thomas Obisesan ${ }^{3}$,

Charmaine Royal ${ }^{4}$, Norman Relkin ${ }^{5}$, L. Adrienne Cupples ${ }^{6}$, Clara Chen ${ }^{6}$, Robert C. Green ${ }^{7},{ }^{1}$ University of Michigan School of Public Health, Ann Arbor, MI, USA; ${ }^{2}$ Case Western Reserve University, Cleveland, OH, USA; ${ }^{3}$ Howard University, Washington, DC, USA; ${ }^{4}$ Duke University, Durham, NC, USA; ${ }^{5}$ Weill Medical College of Cornell University, New York, NY, USA; ${ }^{6}$ Boston University School of Public Health, Boston, MA, USA; ${ }^{7}$ Boston University School of Medicine, Boston, MA, USA.Contacte-mail: jscottr@umich.edu

Background: The $\epsilon 4$ allele of the apolipoprotein E (APOE) gene increases risk for $\mathrm{AD}$ but is neither necessary nor sufficient to cause the disease. APOE genotype disclosure serves as an instructive paradigm regarding the communication of personalized genetic risk information. Our previous work suggested that APOE genotype information can be safely disclosed to first-degree relatives via a traditional genetic counseling protocol. In this study, we compared our original protocol to a briefer, condensed protocol (CP). Methods: Our design was a four-site randomized clinical trial, where participants were a racially diverse sample (mean age $=58$ years, $70 \%$ female, 19\% African American) of first-degree relatives of people with AD. All participants received AD risk information (lifetime risk range: 13-78\%) that incorporated APOE genotype. Participants in the extended protocol (EP) underwent two pre-disclosure counseling sessions (estimated clinician time $=55$ minutes), while participants in the condensed protocol (estimated clinician time $=13$ minutes) received an educational brochure in lieu of one counseling session. Multivariate analyses examined protocol differences in outcome measures assessed at 6 weeks, 6 months and one year following risk disclosure, including: 1) psychological impact (depression, anxiety, and test-specific distress); 2) recall of risk information; and 3) health behavior changes following risk disclosure (e.g., diet, exercise). Results: On average, CP participants experienced similarly low levels of depression and anxiety following risk disclosure as their extended protocol counterparts. CP participants had higher mean levels of test-specific distress at 6 weeks, but not at 6 months or one year. There were no significant differences between the two groups in their recall of APOE genotype, but $\mathrm{CP}$ participants had superior recall of lifetime risk information at 6 weeks $(\mathrm{OR}=1.8, \mathrm{p}=.03)$. The groups did not differ on health behavior changes to reduce risk of AD. Conclusions: As compared to our extended protocol, 
a condensed protocol for communicating genetic risk information for $\mathrm{AD}$ achieved similar results on key study outcomes while requiring significantly less clinician time. Empirically validated alternatives to traditional, time-intensive genetic counseling protocols may be useful in conveying information on susceptibility for common, complex diseases such as Alzheimer's disease.

\section{P2-283 INDIANAPOLIS DISCOVERY NETWORK FOR DEMENTIA: WHERE WE ARE AFTER TWO} YEARS

Ann Marie Hake ${ }^{1}$, Stephanie Munger ${ }^{1}$, Martin R. Farlow ${ }^{1}$, Patrick J. Healey ${ }^{2}$, Mary G. Austrom ${ }^{1}$, Malaz Boustani ${ }^{1},{ }^{1}$ Indiana University School of Medicine, Indianapolis, IN, USA; ${ }^{2}$ St. Vincent Center for Healthy Aging, Indianapolis, IN, USA. Contact e-mail: ahake@iupui.edu

Background: Despite numerous quality improvement efforts, best practice guidelines, and clinical and basic research activities over the past three decades, dementia is still underdiagnosed and undertreated, especially among minorities. Furthermore, even among those receiving optimal care, only a small proportion participate in clinical trials. The Institute of Medicine has recommended the need for system thinking and integrated, locally sensitive collaboration among the various members of the local community, health care systems and research organizations. It is hypothesized that an effective collaboration would improve diagnosis and treatment of the dementia patient, as well as increase participation in research. Methods: A local and diverse network of dementia researchers, clinical providers, and policy makers who are interested in improving the care for dementia patients in Indianapolis was invited to participate in bimonthly meetings. The Network uses the Complex Adaptive System theoretical framework and the Reflective Adaptive Process to facilitate and sustain effective interactions among its members. Results: The Network was established in February 2006 and includes more than 60 members from more than 20 local organizations representing geriatrics, neurology, psychiatry, pathology, neuropsychology, psychology, nursing, social work, primary care, geriatric pharmacy, health services research, epidemiology, pharmaceutical industry, Alzheimer advocacy, state policy makers, health administration, medical education, and biostatistics. The network uses two types of communication among its members. The first is a 3-hour face-to-face bimonthly meeting that includes all members. The second is a project-based workgroup meeting that focuses on a specific task and includes a segment of the network members. To date, the network has built a web-based resource center, completed 4 full-day educational seminars, received funding to test a research enrollment process utilizing the local Alzheimer's Association help line, enabled clinicians to refer patients directly to a research registry, facilitated the submission of two NIH applications, tested a new group interaction method called "consultancy", and has established a new dementia care clinic at the county hospital. Conclusions: Building a local interdisciplinary "think-tank" network in dementia facilitates conducting various collaborative research, educational and quality improvement programs that meet the local research, clinical, and community needs relevant to dementia.

P2-284 INTRODUCING A METHODOLOGY TO USE ACTIVITIES OF DAILY LIVING OUTCOMES IN THE ASSESSMENT OF THE COSTEFFECTIVENESS OF ALZHEIMER'S DISEASE TREATMENTS: A CASE STUDY USING RIVASTIGMINE PATCH

Balázs Nagy ${ }^{1}$, Alan Brennan ${ }^{1}$, Agnes Brandtmuller ${ }^{1}$, Simu Thomas ${ }^{2}$, Meghan Gallagher ${ }^{2}$, Sean D. Sullivan ${ }^{3}$, Ron Akehurst ${ }^{1},{ }^{1}$ University of Sheffield, Sheffield, United Kingdom; ${ }^{2}$ Novartis Pharmaceuticals Corporation, East Hanover, NJ, USA; ${ }^{3}$ University of Washington, Seattle,WA, USA. Contact e-mail: b.nagy@sheffield.ac.uk
Background: Measures of activities of daily living (ADL), as a functional assessment, may be a more accurate payer-relevant reflection of disease burden than cognitive assessments in Alzheimer's disease. The objective of this study is to evaluate the usefulness of ADL instruments in costeffectiveness modelling in Alzheimer's disease. Methods: A core economic model used Mini Mental State Examination (MMSE) scores from the IDEAL trial, which demonstrated significant benefits for rivastigmine patch over placebo. The model was adapted to include Alzheimer's Disease Cooperative Study Activities of Daily Living Inventory (ADCS-ADL) scores, with assumptions made on disease progression over a 5-year period. The clinical pathway was populated based on the results of the pivotal IDEAL trial, with 12-month follow-up data from patients who received rivastigmine patch $(n=383)$ and 6-month follow-up data from patients who received placebo $(n=282)$. The progression of the disease was modelled beyond the study period of one year by defining ADCS-ADL as a function of MMSE, and using published equations on disease progression to predict the natural decline of MMSE. The ADCS-ADL data were then mapped to the Townsend Activities of Daily Living (Townsend-ADL) scale for the prediction of institutionalization. Costing variables included drugs, clinical monitoring, institutionalization and community burden of care. Results: The model based on MMSE scores showed an incremental cost per quality adjusted life year (QALY) gained over best supportive care (BSC) of $£ 13,042$, whereas the Townsend-ADL model indicated total cost savings, primarily attributable to number of institutionalized days avoided over 5 years (67.5 days for ADL compared to 19.2 days for MMSE). Results were insensitive to the type of regression analyses (linear versus logistic) that were carried out to establish the relationship of MMSE and ADL scales. Conclusions: Both the MMSE and Townsend-ADL models demonstrated the cost-effectiveness of rivastigmine patch for patients with $\mathrm{AD}$. ADL modeling should be regarded as a valuable technique for future economic evaluations in the treatment of $\mathrm{AD}$.

\section{P2-285 OLDER ADULTS WITH MEMORY COMPLAINTS CAN ADHERE SUCCESSFULLY TO A HOME- BASED PHYSICAL ACTIVITY PROGRAM: SIX- MONTH RESULTS FROM THE FABS TRIAL}

Nicola T. Lautenschlager ${ }^{1,2}$, Osvaldo P. Almeida ${ }^{1,2}$, Leon Flicker ${ }^{1,3}$, Kathryn R. Greenop ${ }^{1}$, Jacqueline $\mathrm{Ash}^{3,2}$, Kay L. Cox ${ }^{3,1},{ }^{1}$ WA Centre for Health \& Ageing, University of Western Australia, Perth, WA, Australia; ${ }^{2}$ School of Psychiatry and Clinical Neurosciences, University of Western Australia, Perth, WA, Australia; ${ }^{3}$ School of Medicine and Pharmacology, University of Western Australia, Perth, WA, Australia. Contacte-mail: nicola.lautenschlager@uwa.edu.au

Background: Physical activity (PA) has been identified as a potential protective factor for older adults at increased risk for cognitive decline. FABS is a RCT conducted with older volunteers who complain of memory problems. It investigated whether a 6-month individualised home-based PA and behavioural intervention package can significantly affect physical activity levels and cognitive performance. This paper reports the adherence and acceptance of the intervention. Methods: Participants with memory complaints with or without objective cognitive impairment were recruited from the community and randomly assigned to a control or a PA group. The PA group were given an individualised exercise program aimed at achieving 150 minutes/week of moderate exercise. Participants received newsletters, regular phone calls as well as a manual and calendars to help with adherence to the program. All participants wore a pedometer for 1 week at baseline and at 6 months to assess PA. Results: One hundred and seventy participants (84 men, 86 women) mean age 68.5 ( \pm SD 8.6) years started the study. There was no significant difference between groups in the number of participants who were retained in the study, $91 \%$ and $94 \%$ for the PA and control group respectively. Adherence to the prescribed PA program was $78(95 \% \mathrm{CI}, 72.5,83.7) \%$. Men adhered better than women (83.3 $(77.1,89.5) \%$ versus $71.8(62.2,81.3) \% ; \mathrm{p}=0.037)$. There was no difference between individuals with objective cognitive impairment and those without. After 6 months the PA group had significantly higher 